\title{
T-Piece Resuscitator Versus Self-Inflating Bag for Preterm Resuscitation: An Institutional Experience
}

\author{
Archana Jayaram MBBS, Adam Sima MA, Gail Barker RN, and Leroy R Thacker PhD
}

\begin{abstract}
BACKGROUND: Manual ventilation in the delivery room is provided with devices such as selfinflating bags (SIBs), flow-inflating bags, and T-piece resuscitators. OBJECTIVE: To compare the effect of type of manual ventilation device on overall response to resuscitation among preterm neonates born at < 35 weeks gestation. METHODS: Retrospective data were collected in 2 time periods. Primary outcome was overall response to resuscitation, as measured by Apgar score. Secondary outcomes were incidence of air leaks, need for chest compressions/epinephrine, need for intubation, and surfactant use. RESULTS: We identified 294 resuscitations requiring ventilation. SIB was used for 135 neonates, and T-piece was used for 159 neonates. There was no significant difference between the 1-min and 5-min Apgar scores between SIB and T-piece $(P=.77$ and $P=.11$, respectively), nor were there significant differences in secondary outcomes. The rate of rise of Apgar score was higher, by 0.47 , with T-piece, compared to SIB $(95 \%$ CI $0.08-0.87, P=.02)$. CONCLUSIONS: Although some manikin studies favor T-piece for providing reliable and consistent pressures, our experience did not indicate significant differences in effectiveness of resuscitation between the T-piece and SIB in preterm resuscitations. Key words: manual ventilation; selfinflating bag; flow-inflating bag; T-piece; resuscitator; resuscitation; preterm; neonate; Apgar score. [Respir Care 2013;58(7):1233-1236. (C) 2013 Daedalus Enterprises]
\end{abstract}

\section{Introduction}

Manual ventilation during neonatal resuscitation is provided via various devices, such as self-inflating bag (SIB), flow-inflating bag, or T-piece resuscitator. Each device has its own advantages and disadvantages. The SIB, despite its inability to provide CPAP and oxygen reliably, is still used commonly. T-piece resuscitator delivers the desired pressure more accurately, but may have the disadvantage of requiring a longer time to set up, and can deliver hazardous pressures with changes in gas flow. ${ }^{1-4}$

Mr Jayaram and Ms Barker are affiliated with the Division of NeonatalPerinatal Medicine, Children's Hospital of Richmond; and Mr Sima and Dr Thacker are affiliated with the Department of Biostatistics, Virginia Commonwealth University, Richmond, Virginia.

The authors have disclosed no conflicts of interest.

Correspondence: Archana Jayaram MBBS, Division of Neonatal-Perinatal Medicine, Children's Hospital of Richmond, Virginia Commonwealth University, PO Box 980276, Richmond VA 23298-0276.

DOI: $10.4187 /$ respcare.02109
Studies on manikins indicated that T-piece resuscitator guarantees reliable pressure, irrespective of operator-dependent variables. Although studies have compared the 3 devices on manikins, there is not much literature comparing the T-piece resuscitator with other devices during neonatal resuscitations. The objective of this study was to test the hypothesis that premature infants $(<35$ weeks gestational age) resuscitated with T-piece have better response to manual ventilation, as demonstrated by better Apgar scores, compared to those resuscitated with SIB.

\section{Methods}

This study was approved by the institutional review board of Children's Hospital of Richmond, Virginia Commonwealth University.

\section{Subjects}

Retrospective data were collected from the database of the neonatal intensive care unit (NICU) at Children's Hospital of Richmond, Richmond, Virginia. The T-piece resuscitator (Neopuff, Fisher \& Paykel Healthcare, Auck- 


\section{T-Piece Resuscitator Versus Self-Inflating Bag for Preterm Resuscitation}

land, New Zealand) was introduced at our facility for neonatal resuscitations in April 2009, prior to which SIB with manometer was used for all neonatal resuscitations. Due to emerging data from manikin studies regarding potential advantages of providing consistent pressures, ${ }^{1,2,4}$ the decision was made to switch to the T-piece in the delivery rooms and during transport from the delivery room to the NICU. Allowing for a transition period of 6 months wherein all providers attending the delivery room were trained in the use of the T-piece, all resuscitations of NICU admissions between September 2009 and August 2011 were included.

A similar number of records were reviewed in the period prior to the introduction of the T-piece (January 2007 through January 2009). The inclusion criteria consisted of NICU admission, $<35$ weeks gestational age, and requiring manual ventilation in the delivery room. Neonates born with congenital and lethal abnormalities were excluded. There were 7 and 6 delivery room deaths due to unsuccessful resuscitation during the SIB and T-piece periods, respectively. During the SIB period, no definite pressure guidelines were in place. The pressure required to see a response in heart rate and chest rise were used, based on the compliance felt by the provider. There were "default" pressure guidelines in place for the T-piece resuscitator (peak inspiratory pressure of $20 \mathrm{~cm} \mathrm{H}_{2} \mathrm{O}$ and PEEP of $5 \mathrm{~cm} \mathrm{H}_{2} \mathrm{O}$ ), and these could be altered by the provider, based on the response to resuscitation.

During both periods, a respiratory therapist, a neonatal nurse practitioner, or a neonatal fellow/neonatologist was responsible for providing manual ventilation. The Neonatal Resuscitation Program 5th edition guidelines for resuscitation and oxygen use were followed in both time periods. Per the recommendations at that time, "color" was assessed by the resuscitation team to guide supplemental oxygen provision. Although saturation monitors were available for use starting in mid-2010, their use was sporadic and random until definite recommendations came out in the 6th edition of the Neonatal Resuscitation Program (2011). During both periods, all infants $<30$ weeks gestational age were intubated in the delivery room and received prophylactic surfactant, per unit policy at that time. Those $>30$ weeks gestational age were intubated only if the clinical status necessitated intubation.

\section{Measures}

A neonate's initial status and response to resuscitation was measured with the Apgar score, at $1 \mathrm{~min}$ and $5 \mathrm{~min}$ after birth. The gestational age, birth weight, and sex of the neonate were included to account for any Apgar score changes due to these biologic factors. The use of antenatal steroids (either complete or incomplete course) and type of delivery were also recorded.

\section{QUICK LOOK}

\section{Current knowledge}

During neonatal resuscitation, manual ventilation can be provided with a self-inflating bag, a flow-inflating bag, or a T-piece resuscitator. Data are lacking on differences in patient outcomes with these devices.

\section{What this paper contributes to our knowledge}

In a series of 294 neonatal resuscitations there were no significant differences in the effectiveness of resuscitation between a T-piece resuscitator and a self-inflating bag. Apgar scores at 5 min were similar, and there were no differences in the incidence of air leaks.

Due to the high correlation between gestational age and birth weight, a surrogate variable was created that reflects the physical development of each neonate. This score, termed the physical development score, is a summation of the standardized gestational age and birth weight variables. A large positive value of the score indicates higher physical development (present with older, heavier babies), while a large negative value indicates that the neonate is less developed (as seen with younger and smaller babies). Accounting for age and body weight in this manner permits a larger sample size to be used for the comparison of the manual ventilation devices while adjusting for the expected effects of these variables on the Apgar score.

Secondary outcomes analyzed were: occurrence of air leaks (pneumothorax, pneumomediastinum, pulmonary interstitial emphysema) within 24 hours of birth; need for chest compressions/epinephrine in the delivery room; need for intubation in the delivery room; and surfactant use in neonates between 30 and 35 weeks gestational age, as it was our unit guideline to give prophylactic surfactant to all neonates $<30$ weeks gestational age.

\section{Statistical Analysis}

All variables are summarized as mean $\pm \mathrm{SD}$ or frequency and percentage, separately for each cohort. Independent $t$ test or the Pearson test of association was performed to determine marginal differences between the variables. A linear mixed-effects model was used to test the primary hypothesis with the physical development score, sex, delivery type, antenatal steroids use, time, and the interaction of the breathing device with each of these variables. This model explicitly models the mean Apgar score at each time point for each device. Using this information, the mean rate of increase of the mean Apgar score can be calculated and compared for each device. The same 


\section{T-Piece Resuscitator Versus Self-Inflating Bag for Preterm Resuscitation}

Table. Summary Statistics of Variables $(P<.05$ reflects statistical significance)

\begin{tabular}{|c|c|c|c|c|c|c|}
\hline & \multicolumn{3}{|c|}{ 23-35 Weeks Gestational Age } & \multicolumn{3}{|c|}{ 30-35 Weeks Gestational Age } \\
\hline & $\begin{array}{c}\text { Self-Inflating Bag } \\
\quad n=135\end{array}$ & $\begin{array}{c}\text { T-piece } \\
n=159\end{array}$ & $P$ & $\begin{array}{c}\text { Self-Inflating Bag } \\
n=48\end{array}$ & $\begin{array}{l}\text { T-piece } \\
n=75\end{array}$ & $P$ \\
\hline \multicolumn{7}{|l|}{ Apgar Score, mean $\pm \mathrm{SD}$} \\
\hline At $1 \mathrm{~min}$ & $4.2 \pm 1.9$ & $4.0 \pm 2.3$ & .35 & $4.2 \pm 1.7$ & $4.3 \pm 2.3$ & .80 \\
\hline At $5 \mathrm{~min}$ & $6.0 \pm 1.9$ & $6.2 \pm 1.8$ & .26 & $6.4 \pm 1.8$ & $6.7 \pm 1.6$ & .28 \\
\hline Gestational age, mean $\pm \mathrm{SD}$ weeks & $28.2 \pm 3.2$ & $28.8 \pm 3.6$ & .13 & $31.9 \pm 1.1$ & $32.1 \pm 1.5$ & .32 \\
\hline Birth weight, mean $\pm \mathrm{SD} g$ & $1,152 \pm 512$ & $1,299 \pm 603$ & .03 & $1,674 \pm 413$ & $1,798 \pm 449$ & .13 \\
\hline Female, no. (\%) & $66(48.9)$ & $62(39.2)$ & .10 & $24(50.0)$ & $28(37.3)$ & .17 \\
\hline Delivery via C-section, no. (\%) & $84(62.2)$ & $103(64.8)$ & .65 & $27(56.3)$ & $52(69.3)$ & .14 \\
\hline Received antenatal steroids, no. (\%) & $73(54.1)$ & $128(81.5)$ & $<.001$ & $24(50.0)$ & $54(74.0)$ & .007 \\
\hline Received chest compressions or epinephrine, no. (\%) & $8(5.9)$ & $16(10.1)$ & .20 & $1(2.1)$ & $5(6.7)$ & .25 \\
\hline Air leak, no. (\%) & $8(5.9)$ & $5(3.1)$ & .25 & $0(0.0)$ & $1(1.3)$ & .42 \\
\hline Intubated, no. $(\%) *$ & & & & $25(52.1)$ & $28(37.3)$ & .11 \\
\hline Received surfactant, no. $(\%)^{*}$ & & & & $12(25.0)$ & $11(14.7)$ & .15 \\
\hline
\end{tabular}

* All infants $<30$ weeks were intubated and received prophylactic surfactant, per unit guidelines.

predictor variables, except for time, were used in a logistic regression with the secondary response variables of air leaks, chest compressions/epinephrine, surfactant use and intubation. Statistics software (SAS, SAS Institute, Cary, North Carolina) was used for all analyses. A type-1 error rate of .05 was used for all analyses.

\section{Results}

A total of 294 neonates were identified. SIB was used for 135 neonates, and T-piece was used for 159 neonates. Of the 123 who were between 30 and 35 weeks gestational age, 48 were resuscitated with SIB, and 75 with T-piece. The mean Apgar scores at $1 \mathrm{~min}$ and 5 min were 4.2 and 6.0 for the SIB group, and 4.0 and 6.2 for the T-piece group. A summary of the variables is presented in the Table. The T-piece group was more likely to have received antenatal steroids and was slightly heavier than the SIB group. No other statistically significant differences were observed between the groups. Four observations (sex and 5-min Apgar score from 2 different neonates, and antenatal steroids use in 2 neonates) were excluded from the analysis due to missing covariates.

Neonates resuscitated with T-piece showed a 2.23 point (standard error $0.14,95 \%$ CI $1.96-2.50$ ) increase from the 1 -min to the 5 -min Apgar score $(P<.001)$, while those resuscitated with SIB had an Apgar score increase of 1.76 points (standard error $0.15,95 \%$ CI $1.47-2.04$ ) between the 2 time points $(P<.001)$. This results in a 0.47 (standard error $0.20,95 \%$ CI $0.08-0.87$ ) higher increase for the T-piece group than the SIB group $(P=.02)$. The Apgar scores were not different between the devices at either $1 \mathrm{~min}$ or $5 \min (P=.77$ and $P=.11$, respectively $)$ (Figure).

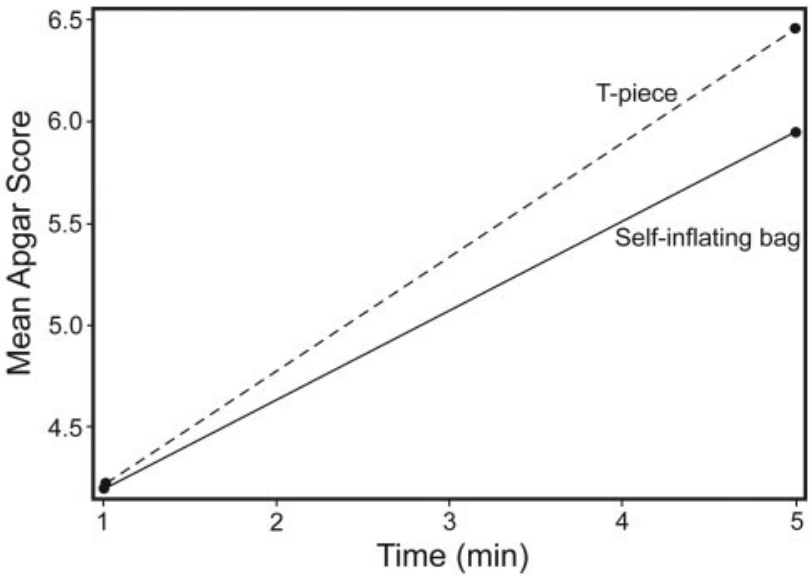

Figure. Apgar scores at $1 \mathrm{~min}$ and $5 \mathrm{~min}$ with T-piece versus self-inflating bag, in neonates 23-35 weeks gestational age. The Apgar score rose faster with T-piece, but the difference was not statistically significant $(P=.11)$.

The use of antenatal steroids also had an effect on the overall mean Apgar scores, which was moderated by the breathing device. The mean Apgar score was 0.67 points (standard error $0.30,95 \%$ CI $0.07-1.27$ ) higher in the neonates who received antenatal steroids in the SIB group $(P=.03)$ than in those who did not receive steroids. No such difference was observed between the neonates who received and did not receive antenatal steroids in the $\mathrm{T}$ piece group $($ difference $=0.32$, standard error $0.39,95 \% \mathrm{CI}$ -0.45 to $1.09, P=.42$ ). There were no differences observed between the SIB and T-piece devices with respect to the overall mean, sex, and delivery type. The physical development score for each neonate was an important predictor of the 1-min and 5-min Apgar scores, in that older 


\section{T-Piece Resuscitator Versus Self-Inflating Bag for Preterm Resuscitation}

and heavier neonates had higher Apgar scores at each time point, and this effect was the same for both groups. Furthermore, the secondary analyses revealed no significant differences between devices for any of the response variables.

\section{Discussion}

The T-piece resuscitator, although it offers the theoretical advantage of being able to provide consistent PEEP, as proven by manikin studies, ${ }^{2,4,5}$ has insufficient evidence from neonatal studies to attest to this benefit. ${ }^{6}$ It appears that widespread use of PEEP during neonatal resuscitation is derived from animal models and observational studies. ${ }^{7,8}$ In this study, T-piece increased the mean 1-min to 5-min Apgar score more rapidly than SIB. Although a statistically significant difference in rate of rise was seen between the 2 devices, since the Apgar score at $5 \mathrm{~min}$ were not found to be different, the conclusion that the T-piece is superior cannot be definitively stated. The observation of a more rapid rate of rise in the T-piece group may indicate that this group could perhaps demonstrate a superior outcome at some point beyond $5 \mathrm{~min}$. Neonates who did not receive antenatal steroids and were resuscitated with SIB had a lower Apgar score than those who received both SIB and antenatal steroids. This decrease based on antenatal steroids was not observed in the neonates who were resuscitated with the T-piece. We can only speculate that this observation may be due to more consistent delivery of PEEP with the T-piece resuscitator and therefore better maintenance of functional residual capacity, even in the absence of antenatal steroid benefit.

The secondary analyses indicated that there were no differences between the devices for the combined incidence of air leaks occurring within 24 hours of life, or the need for use of chest compressions or epinephrine in the delivery room. Among neonates with gestational age between 30 and 35 weeks, neither surfactant use nor need for intubation in the delivery room showed any differences between the devices. Our results support similar findings by Dawson et al, ${ }^{1}$ who compared 5 -min $\mathrm{S}_{\mathrm{pO}_{2}}$ in preterm infants resuscitated with SIB or T-piece, and did not find any significant difference. ${ }^{5}$

A limitation of this study is the retrospective nature using a convenience sample, and therefore the lack of randomization, which makes it difficult to control for operator experience. Since this is a retrospective, single-center study, it also makes it difficult to generalize the results. This study was designed primarily to look at differences in overall Apgar scores, which has some limitations for use in extremely preterm neonates, ${ }^{9}$ but is widely used nonetheless. Instead, an improvement in heart rate may have been a better indicator of response to resuscitation. It would also have been useful to examine the effectiveness of resuscitation by analyzing Apgar scores beyond $5 \mathrm{~min}$, as the faster rise that was observed with T-piece may have been more obvious and shown a significant difference. Because of the infrequency of outcomes such as air leaks and cardiopulmonary resuscitation in the delivery room, the study was most likely underpowered for many of the secondary analyses.

\section{Conclusions}

Although the T-piece resuscitator offers advantages over SIB in the ability to provide continuous distending pressure and consistent pressures, in our experience manual ventilation in the delivery room is feasible with both SIB and T-piece resuscitator with no significant difference in effectiveness of resuscitation or in immediate clinical outcomes. If future recommendations are to clearly favor one device over the other, larger randomized neonatal studies comparing the use of different devices for manual ventilation during neonatal resuscitation are needed.

\section{REFERENCES}

1. Dawson JA, Gerber A, Kamlin CO, Davis PG, Morley CJ. Providing PEEP during neonatal resuscitation: which device is best? J Paediatr Child Health 2011;47(10):698-703.

2. Bennett S, Finer NN, Rich W, Vaucher Y. A comparison of three neonatal resuscitation devices. Resuscitation 2005;67(1):113-118.

3. Hawkes CP, Oni OA, Dempsey EM, Ryan CA. Potential hazard of the Neopuff T-piece resuscitator in the absence of flow limitation. Arch Dis Child Fetal Neonatal Ed 2009;94(6):F461-F463.

4. Klingenberg C, Dawson JA, Gerber A, Kamlin CO, Davis PG, Morley CJ. Sustained inflations: comparing three neonatal resuscitation devices. Neonatology 2011;100(1):78-84.

5. Dawson JA, Schmölzer GM, Kamlin CO, Te Pas AB, O’Donnell CP, Donath SM, et al. Oxygenation with T-piece versus self-inflating bag for ventilation of extremely preterm infants at birth: a randomized controlled trial. J Pediatr 2011;158(6):912-918.e1-e2.

6. O'Donnell C, Davis P, Morley C. Positive end-expiratory pressure for resuscitation of newborn infants at birth. Cochrane Database Syst Rev 2004;(4):CD004341.

7. Probyn ME, Hooper SB, Dargaville PA, McCallion N, Crossley K, Harding R, Morley CJ. Positive end expiratory pressure during resuscitation of premature lambs rapidly improves blood gases without adversely affecting arterial pressure. Pediatr Res 2004;56(2):198204.

8. Upton CJ, Milner AD. Endotracheal resuscitation of neonates using a rebreathing bag. Arch Dis Child 1991;66(1 Spec No):39-42.

9. Hegyi T, Carbone T, Anwar M, Ostfeld B, Hiatt M, Koons A, et al. The Apgar score and its components in the preterm infant. Pediatrics 1998;101(1 Pt 1):77-81. 